ISSN 1392-3196 / e-ISSN 2335-8947

Zemdirbyste-Agriculture, vol. 104, No. 2 (2017), p. 147-156

DOI 10.13080/z-a.2017.104.019

\title{
Genetic behaviour of qualitative and seed yield-related traits in Brassica rapa
}

\author{
Aamer MUMTAZ ${ }^{1,2}$, Hafeez Ahmad SADAQAT ${ }^{2}$, Muhammad SAEED ${ }^{1}$, \\ Muhammad Irfan YOUSAF ${ }^{1,2}$, Aamar SHEHZAD ${ }^{2}$, Hafiz Ghulam Moh Din AHMED ${ }^{2}$ \\ ${ }^{1}$ Maize and Millets Research Institute \\ Yousafwala, Sahiwal, Pakistan \\ E-mail: aamer3002@gmail.com \\ ${ }^{2}$ University of Agriculture \\ Faisalabad 47000, Pakistan
}

\begin{abstract}
The domestic production of edible oil meets only $20.23 \%$ of the demand in Pakistan, and domestic oil production needs to be increased. Thanks to its short life cycle, high yield and yellow seed colour, oilseed rape (Brassica rapa L.) is a potentially useful source to fill the gap between production and consumption. This study was designed to ascertain the genetic expression of descriptive and seed yield-related traits in four $B$. rapa accessions (UAF11, Toria, BSA and TP-124-1) and their hybrid progenies obtained from complete diallel mating crossings. Heterosis and heterobeltiosis were computed for these traits. Seed colour was varying shades of brown and showed Mendelian genetics. Seed shape was consistently round in all lines, and leaf hairiness was a characteristic of all crosses including self. Each parent line had a particular leaf shape as elliptic, pandurate, lanceolate and ovate, and no intermediate or new shapes were observed in $F_{1}$. Leaf colour was varying shades of green. Seed shape, leaf shape and leaf colour were under digenic control with no epistatic or maternal effects. Leaf shape showed multiple allelism. Seed shape showed no variations. Leaf hairiness was dominant and under monogenic control. Number of siliqua/primary branches of plant, number of siliqua/secondary branches of plant and total number of siliqua/plant directly affect seed yield of plant while effect of plant height is indirect. Variability was observed in heterosis and heterobeltiosis for all traits. UAF-11 and its crosses with Toria and TP-124-1 and their reciprocals were identified as the best hybrid progenies for future breeding efforts. Our results for the traits, compared here will, hopefully, facilitate future efforts to select productive $B$. rapa accessions for breeding programs aimed at optimizing heterosis.
\end{abstract}

Key words: heterobeltiosis, heterosis, leaf hairiness, seed colour, seed shape.

\section{Introduction}

The domestic production of edible oil is below current demand in Pakistan, where the oilseed requirement was 2.748 million tons during the year 2014-2015. Local production of oilseed was estimated at around 0.556 million tons, i.e. $20.23 \%$ of the total demand. Because of this shortfall, Pakistan imported 1.738 million tons of oil at the cost of PKR 269.412 billion (USD 2.663 billion) in 2014-2015. The local production of rapeseed oil during this period was 0.067 million tons, which accounted for about $12 \%$ of the total oilseed production. According to an estimate of industrial usage (cosmetics, paints and other products), domestic production was able to meet only $10 \%$ of the total need nationwide. About 0.2 million tons of oil are exported to Afghanistan, although this amount does not include the smuggled oil (Government of Pakistan, 2015-2016).
The oilseed sector needs immediate attention due to rising demand because of the increasing human population and plant crop usage. Rapeseed and mustard (Brassica oilseeds) crops are the second largest source of vegetable oil after cottonseed in Pakistan. In 2014 $2015,0.533$ million hectares were devoted to rapeseed cultivation, while 9.260 million hectares were sown with wheat and 2.748 million hectares were devoted to rice. For other local sources of edible oil (e.g., sunflower and soybean) the share of arable land used to grow these crops is very low (Government of Pakistan, 2015-2016).

Brassica rapa (syn. campestris), commonly known as rapeseed or desi sarson, is an important oilseed crop worldwide. This species belongs to the order Brassicales and the family Brassicaceae. Oil content ranges from 35\% to 46\% (Kumar et al., 2011; Mumtaz

Please use the following format when citing the article:

Mumtaz A., Sadaqat H. A., Saeed M., Yousaf M. I., Shehzad A., Ahmed H. G. M. D. 2017. Genetic behaviour of qualitative and seed yield-related traits in Brassica rapa. Zemdirbyste-Agriculture, 104 (2): 147-156 DOI 10.13080/z-a.2017.104.019 
et al., 2014), but low percentage yields of high-quality oil are a recurring problem. Existing $B$. rapa varieties are low yielding and have a maturity period of 100 to 140 days. Aphid attack, white rust of cruciferous vegetables and shattering when ripe are the main problems that threaten yields. Lodging due to wind and rain is also common. There is thus a critical need to develop varieties with seed yields comparable in economic value to wheat, and which mature as early as in 60 to 90 days to suit local cropping systems.

Farmers can be hesitant to try different accessions because of concerns about economic return, but if varieties with a short life cycle and high yield are made available, they may be attractive enough for farmers to plant in order to increase production with time. Yellow seeded varieties of $B$. rapa reportedly have higher oil yields (Kumar et al., 2011), so high-yielding yellow seeded varieties should be bred to increase oil yields. Seed yield is a complex trait influenced by several components, especially seed weight, siliquae per plant, seeds per siliqua and numbers of locules per siliqua (Sinhamahapatra et al., 2010).

Seed colour is thought to be controlled by monogenic (Rather et al., 2012; Rahman, McVetty, 2011), digenic (Vera et al., 1979; Stringam, 1980), trigenic (Mohammad et al., 1942) or quadrigenic inheritance (Zhang et al., 2009). Leaf hairiness is thought to be monogenically controlled (Rahman, 2014). However, current knowledge regarding maternal effects and heterosis effects is incomplete (Mumtaz et al., 2015).

The present research was done in an effort to gather and systematize information on the genetics of different qualitative and seed yield related traits in $B$. rapa. Our findings will help dispel confusion and support efforts by researchers to improve B. rapa seedrelated traits and oil quality. We also hope that new knowledge gained here on the growth characteristics and yields of different accessions will help convince farmers to improve agricultural practices and hence oilseed production.

\section{Material and methods}

The present research was conducted at the research area of the Department of Plant Breeding and
Genetics, University of Agriculture, Faisalabad, Pakistan during 2013-2014. Four accessions of oilseed rape (Brassica rapa L.) were sown: UAF-11, BSA, Toria and TP-124-1. They were crossed in complete diallel mating design in all possible combinations including self and reciprocal crosses. All agronomic practices recommended for B. rapa were followed throughout the study. All necessary precautions were taken to avoid contamination of the genetic material at the time of crossing.

Traits studied. Data were collected on qualitative (seed coat colour, seed shape, leaf hairiness, leaf shape, leaf colour and plant height), plant-related (plant height) and seed yield-related traits (seed volume, 1000 seed weight, number of siliquae/primary branches of plant, number of siliquae/secondary branches of plant, total number of siliquae/plant, seed yield/plant, siliqua length and number of seeds/siliqua).

Experimental design. Twelve hybrid progenies along with their parents were sown during November 2013 in the field. The design used for these experiments was a randomized complete block design.

Statistical analysis. The effects of heterosis and heterobeltiosis were estimated according to the method published by Falconer and Mackay (1996) and correlation was calculated according to Mead et al. (2002) with the software XLSTAT.

\section{Results}

Descriptive qualitative traits. Seed colour. In this study, we observed that seed colour was under digenic control, as intermediate dominance indicated the presence of two genes. Intermediate dominance was evident in all B. rapa crosses as a variety of shades of brown colour (Tables 1-2). Black seeded Toria and yellow seeded UAF-11 produced very light brown seed, black seeded Toria and dark brown seeded BSA produced light brown seed, black seeded Toria and dark brown seeded TP-124-1 produced dark brown seed, dark brown seeded BSA and yellow seeded UAF-11 produced light brown seed, dark brown seeded BSA and dark brown seeded TP-124-1 produced dark brown seed, dark brown seeded TP-214-1, and yellow seeded UAF-11 produced light brown seed. The results of reciprocal crosses were the same.

Table 1. Qualitative traits inheritance in Brassica rapa

\begin{tabular}{|c|c|c|c|c|}
\hline $\begin{array}{l}\text { Serial } \\
\text { No. }\end{array}$ & $\begin{array}{l}\text { Parent and crosses } \\
\text { combinations }\end{array}$ & $\begin{array}{l}\text { Seed } \\
\text { colour }\end{array}$ & $\begin{array}{l}\text { Leaf } \\
\text { shape }\end{array}$ & Leaf colour \\
\hline 1. & BSA & dark brown & pandurate & dark green \\
\hline 2. & $\mathrm{BSA} \times \mathrm{UAF}-11$ & light brown & pandurate & dark green \\
\hline 3. & $\mathrm{BSA} \times$ Toria & light brown & pandurate & medium green \\
\hline 4. & $\mathrm{BSA} \times \mathrm{TP}-124-1$ & dark brown & pandurate & medium green \\
\hline 5. & Toria & black & pandurate & dark green \\
\hline 6. & Toria $\times$ UAF -11 & very light brown & lanceolate & light green \\
\hline 7. & Toria $\times$ BSA & light brown & ovate & dark green \\
\hline 8. & Toria $\times$ TP-124-1 & dark brown & ovate & dark green with purple \\
\hline 9. & TP-124-1 & dark brown & pandurate & light green \\
\hline 10. & TP-124-1 × UAF-11 & light brown & lanceolate & dark green \\
\hline 11. & TP-124-1 × BSA & dark brown & lanceolate & medium green with dark purple \\
\hline 12. & TP-124-1 $\times$ Toria & dark brown & lanceolate & medium green with dark purple \\
\hline 13. & UAF-11 & yellow & elliptic & medium green with purple \\
\hline 14. & $\mathrm{UAF}-11 \times \mathrm{BSA}$ & light brown & pandurate & dark green \\
\hline 15. & UAF-11 × Toria & very light brown & ovate & dark green \\
\hline 16. & UAF-11 × TP-124-1 & light brown & lanceolate & medium green with dark purple \\
\hline
\end{tabular}


Table 2. Digenic inheritance in Brassica rapa

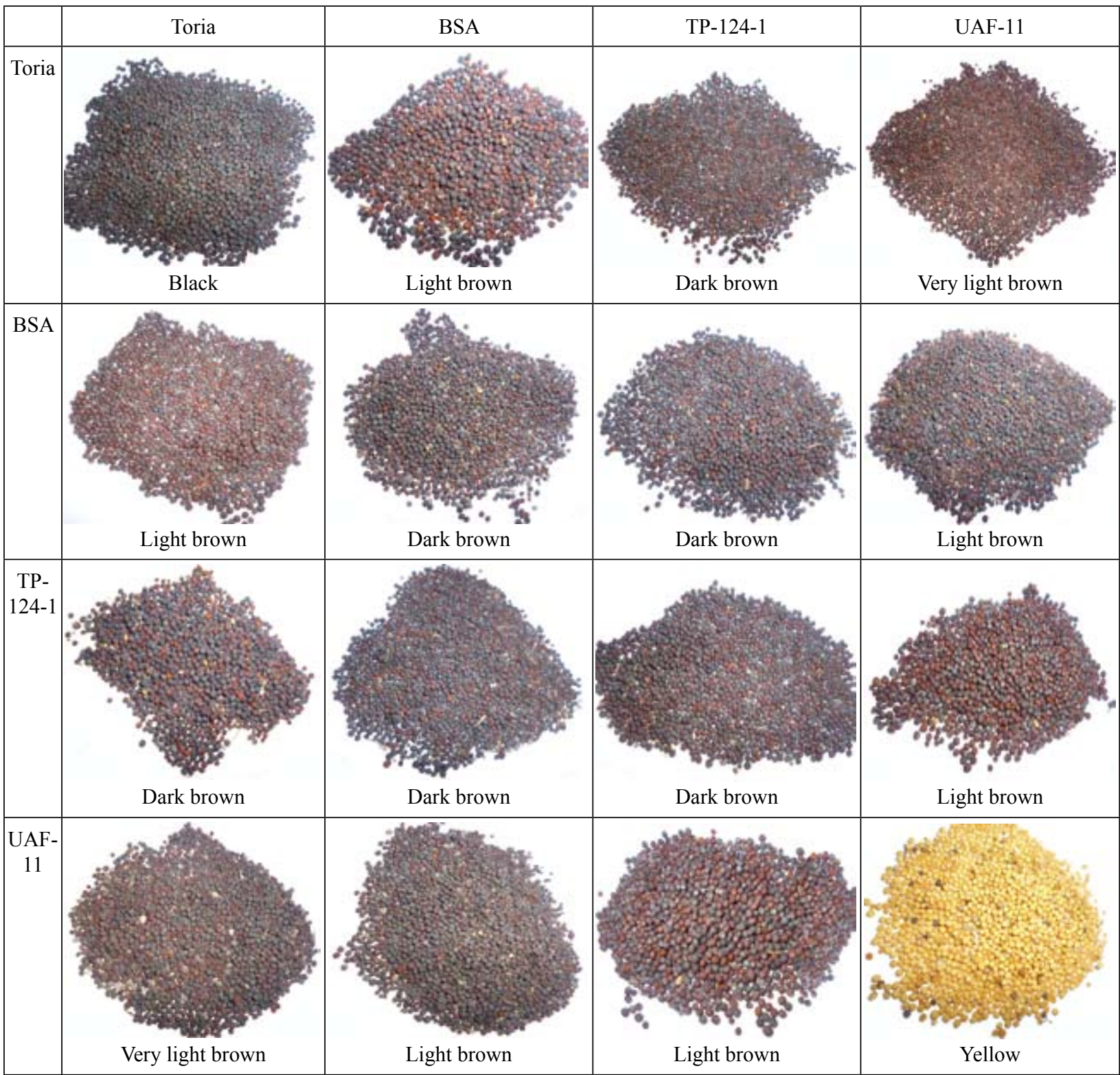

Seed shape. A single shape (round) was observed consistently in all genotypes.

Leaf hairiness. All parents were non-hairy, and in $\mathrm{F}_{1}$, no hairy plants were observed. This clearly indicated that leaf hairiness was under monogenic control, and that hairiness is dominant over non-hairiness.

Leaf shape. Four types of leaf shape were observed in the parents. In $\mathrm{F}_{1}$ no other shapes were seen, which means that intermediate dominance did not affect leaf shape. The pandurate shape was dominant over all other shapes, leanceolate was dominant over ovate, and ovate was dominant over elliptic, which means that elliptic was recessive to all traits.

Leaf colour. Large variations were observed in leaf colour and intensity. Green colour was dominant over purple green, and dark green was dominant over light green. Medium shades of colour had intermediate dominance.

Heterosis and heterobeltiosis. Plant height (cm). Individual mean comparisons of all accessions are reported in the Table 6. Parent BSA had the tallest plant height $(174.9 \mathrm{~cm})$ and parent UAF-11 had the shortest plant height $(93.73 \mathrm{~cm})$. Among hybrid progenies, TP-124-1 $\times$ BSA had the tallest plant height $(136.83 \mathrm{~cm})$ and UAF$11 \times$ TP-124-1 the shortest plant height $(116.75 \mathrm{~cm})$. The estimated heterosis effects ranged from -23.649 (Toria $\times$ BSA) to $15.057(\mathrm{TP}-124-1 \times \mathrm{UAF}-11)$ and estimated heterobeltiosis effects ranged from -20.132 (Toria $\times$ BSA) to 0.914 (TP-124-1 $\times$ UAF-11). Highly significant positive heterosis was observed in one hybrid progeny (TP-124-1 × UAF-11). Significant positive heterosis was observed in two hybrid progenies (UAF-11 $\times$ Toria and UAF-11 $\times$ TP-124-1). Non-significant positive heterosis was observed in one hybrid progeny (Toria $\times$ UAF-11). Highly significant negative heterosis was observed in three hybrid progenies $(\mathrm{BSA} \times$ Toria, BSA $\times \mathrm{TP}-124-$ 1 and Toria $\times$ BSA). Significant negative heterosis was observed in two hybrid progenies (UAF-11 $\times$ BSA and TP-124-1 $\times$ BSA), and non-significant negative heterosis was observed in three hybrid progenies $(\mathrm{BSA} \times \mathrm{UAF}-11$, Toria $\times$ TP-124-1 and TP-124-1 $\times$ Toria).

We found no significant positive heterobeltiosis in any hybrid progeny. Non-significant positive heterobeltiosis was found in only one hybrid progeny 
(TP-124-1 $\times$ UAF-11) and highly significant negative heterobeltiosis was found in all other hybrid progenies except UAF-11 $\times$ TP-124-1, which had non-significant negative heterobeltiosis (Table 3 ).

Seedvolume $\left(\mathrm{cm}^{3}\right)$. Individual mean comparisons of all accessions are shown in the Table 6. Parent TP-1241 had the largest seed volume $\left(7.86 \mathrm{~cm}^{3}\right)$ and parent BSA had the smallest seed volume $\left(5.58 \mathrm{~cm}^{3}\right)$. Among hybrid progenies, TP-124-1 $\times$ BSA had the largest seed volume $\left(7.52 \mathrm{~cm}^{3}\right)$ and BSA $\times$ UAF-11 had the smallest seed volume $\left(5.35 \mathrm{~cm}^{3}\right)$. The estimated heterosis effects ranged from $-16.275(\mathrm{BSA} \times \mathrm{UAF}-11)$ to $11.855(\mathrm{TP}-124-1 \times$ BSA), and estimated heterobeltiosis effects ranged from $-25.694(\mathrm{BSA} \times \mathrm{UAF}-11)$ to $2.431(\mathrm{UAF}-11 \times$ Toria $)$. Highly significant positive heterosis was observed in four hybrid progenies (Toria $\times$ UAF-11, BSA $\times$ TP-124 and their reciprocal crosses). Highly significant negative heterosis was observed in seven hybrid progenies (BSA $\times$ UAF-11, TP-124-1 $\times$ UAF-11, Toria $\times$ TP-124-1, BSA $\times$ Toria and their reciprocal crosses except for Toria $\times$ BSA, which showed non-significant negative heterosis. For heterobeltiosis only two crosses $(\mathrm{BSA} \times \mathrm{UAF}-11$ and its reciprocal cross) showed a highly significant positive increase over their best parent. Nine crosses (BSA $\times$ UAF-11, Toria $\times$ TP-124-1, TP-124-1 $\times$ UAF-11, UAF$11 \times$ Toria) and their reciprocal crosses) showed highly significant negative increases over their best parent, except for Toria $\times$ UAF-11, which had non-significant negative heterobeltiosis (Table 3 ).

1000 seed weight (g). Individual mean comparisons of all accessions are shown-in Table 6 . Parent TP-124-1 had the greatest 1000 seed weight
(4.392 g) and parent BSA had the lowest 1000 seed weight $(2.397 \mathrm{~g})$. Among hybrid progenies, TP-124-1 $\times$ BSA had the greatest 1000 seed weight $(4.808 \mathrm{~cm})$ and UAF-11× BSA had the lowest 1000 seed weight $(2.197 \mathrm{~cm})$. The estimated heterosis effects ranged from -30.353 (UAF$11 \times \mathrm{BSA})$ to $41.651(\mathrm{TP}-124-1 \times \mathrm{BSA})$, and estimated heterobeltiosis effects ranged from -43.834 (UAF-11 $\times$ BSA) to 9.479 (TP-124-1 $\times$ BSA). Highly significant positive heterosis was observed in five hybrid progenies (BSA $\times$ TP-124-1, Toria $\times$ BSA, Toria $\times$ TP-124-1 and TP-124-1 $\times$ BSA and TP-124-1 $\times$ Toria). Non-significant positive heterosis was observed in two hybrid progenies (UAF-11 × TP-124-1 and TP-124-1 × UAF-11). Highly significant negative heterosis was observed in three hybrid progenies $(\mathrm{UAF}-11 \times \mathrm{BSA}, \mathrm{BSA} \times \mathrm{UAF}-11$ and $\mathrm{BSA} \times$ Toria). Significant negative heterosis was observed in one hybrid progeny (Toria $\times$ UAF-11), and nonsignificant negative heterosis was observed in one hybrid progeny (UAF-11 $\times$ Toria). Highly significant positive heterobeltiosis was observed in two hybrid progenies $($ BSA $\times$ TP-124-1 and TP-124-1 $\times$ BSA). Significant positive heterobeltiosis was observed in only one hybrid progeny (Toria $\times$ BSA) and highly significant negative heterobeltiosis was observed in seven hybrid progenies (UAF-11 $\times$ BSA, UAF-11 $\times$ Toria, BSA $\times$ UAF-11, BSA $\times$ Toria, Toria $\times$ UAF-11, Toria $\times$ TP-124- 1 and TP-124-1 $\times$ UAF-11). Non-significant negative heterobeltiosis was observed in none of the hybrid progenies (Table 3 ).

Number of siliquae/primary branches of plant. Individual mean comparisons of all accessions are shown in the Table 6. Parent TP-124-1 had the largest number of siliquae/primary branches of the plant (307.2) and parent

Table 3. Heterosis and heterobeltiosis of plant height, seed volume and 1000 seed weight

\begin{tabular}{|c|c|c|c|c|c|c|}
\hline \multirow{2}{*}{ Hybrid progenies } & \multicolumn{2}{|c|}{ Plant height } & \multicolumn{2}{|c|}{ Seed volume } & \multicolumn{2}{|c|}{1000 seed weight } \\
\hline & heterosis & heterobeltiosis & heterosis & heterobeltiosis & heterosis & heterobeltiosis \\
\hline UAF-11 $\times$ BSA & $-7.29 *$ & $-29.58 * *$ & $-13.67 * *$ & $-23.38 * *$ & $-30.35 * *$ & $-43.83 * *$ \\
\hline UAF-11 × Toria & $8.51 *$ & $-11.82 * *$ & $8.54 * *$ & $2.43 * *$ & $-0.69 \mathrm{~ns}$ & $-12.64 * *$ \\
\hline UAF-11 × TP-124-1 & $10.56^{*}$ & $-3.032 \mathrm{~ns}$ & $-8.03 * *$ & $-11.90 * *$ & $2.37 \mathrm{~ns}$ & $-3.23 *$ \\
\hline $\mathrm{BSA} \times \mathrm{UAF}-11$ & $-3.45 \mathrm{~ns}$ & $-26.66 * *$ & $-16.28 * *$ & $-25.69 * *$ & $-26.83 * *$ & $-41.00 * *$ \\
\hline BSA $\times$ Toria & $-19.46^{* *}$ & $-26.30 * *$ & $-3.43 * *$ & $-9.55 * *$ & $-9.15 * *$ & $-17.91 * *$ \\
\hline $\mathrm{BSA} \times \mathrm{TP}-124-1$ & $-13.53 * *$ & $-27.01 * *$ & $10.86^{* *}$ & $-5.22 * *$ & $40.87 * *$ & $8.87 * *$ \\
\hline Toria $\times$ UAF-11 & $4.41 \mathrm{~ns}$ & $-15.15^{* *}$ & $7.58 * *$ & $1.53 \mathrm{~ns}$ & $-3.71^{*}$ & $-15.29 * *$ \\
\hline Toria $\times$ BSA & $-23.65 * *$ & $-30.13 * *$ & $-0.25 \mathrm{~ns}$ & $-6.57 * *$ & $15.89 * *$ & $4.71 *$ \\
\hline Toria $\times$ TP-124-1 & $-1.20 \mathrm{~ns}$ & $-9.64 * *$ & $-4.98 * *$ & $-13.87 * *$ & $7.88 * *$ & $-9.59 * *$ \\
\hline TP-124-1 $\times$ BSA & $-7.33 *$ & $-21.76^{* *}$ & $11.86^{* *}$ & $-4.37 * *$ & $41.65 * *$ & $9.48 * *$ \\
\hline TP-124-1 × Toria & $-17.28 \mathrm{~ns}$ & $-24.30 * *$ & $-4.56 * *$ & $-13.49 * *$ & $20.54 * *$ & $1.03 \mathrm{~ns}$ \\
\hline TP-124-1 × UAF-11 & $15.06^{* *}$ & $0.91 \mathrm{~ns}$ & $-9.03 * *$ & $-12.85 * *$ & $1.17 \mathrm{~ns}$ & $-4.37 * *$ \\
\hline
\end{tabular}

$*$ - significant, ${ }^{* *}$ - highly significant, $\mathrm{ns}$ - non-significant: $\mathrm{t}_{\text {tab }}$ at $15 \mathrm{df}=2.131(\alpha=0.05), 2.947(\alpha=0.01)$

UAF-11 had the lowest number of siliquae/primary branches of plant (213.5). Among the hybrid progenies, Toria $\times$ BSA had the largest number of siliquae/primary branches of plant (629.9) and Toria $\times$ TP-124-1 had the smallest number of siliquae/primary branches of plant (214). The estimated heterosis effects ranged from -19.062 (Toria $\times$ TP-124-1) to 112.123 (Toria $\times$ BSA), and estimated heterobeltiosis effects ranged from -30.334 $($ Toria $\times$ TP-124-1) to 105.046 (Toria $\times$ BSA). Highly significant positive heterosis was observed in nine hybrid progenies (UAF-11 × BSA, UAF-11 $\times$ Toria, UAF-11 $\times$ TP-124-1, BSA $\times$ Toria, BSA $\times$ TP-124-1, Toria $\times$ UAF11, Toria $\times$ BSA, TP-124-1 $\times$ BSA and TP-124- $\times$ UAF11). Highly significant negative heterosis was observed in three hybrid progenies $(\mathrm{BSA} \times \mathrm{UAF}-11$, Toria $\times$ TP124-1 and TP-124-1 $\times$ Toria). Highly significant positive heterobeltiosis was observed in four hybrid progenies (UAF-11 $\times$ Toria, UAF-11 $\times$ TP-124-1, BSA $\times$ Toria and TP-124-1 $\times$ UAF-11). Significant positive heterobeltiosis was observed in three hybrid progenies (BSA $\times$ TP-1241 , Toria $\times$ UAF-11 and Toria $\times$ BSA). Non-significant positive heterobeltiosis was observed in one hybrid progeny (TP-124-1 × BSA). Highly significant negative heterobeltiosis was observed in two hybrid progenies $(\mathrm{UAF}-11 \times$ BSA and BSA $\times$ UAF-11) (Table 4).

Number of siliquae/secondary branches of plant. Individual mean comparisons of all accessions are shown in Table 6. Parent Toria had the largest number 
of siliquae/secondary branches of the plant (954.6) and parent UAF-11 had the smallest number of siliquae/ secondary branches of plant (183.1). Among the hybrid progenies, BSA $\times$ Toria had the largest number of siliquae/ secondary branches of plant $(1915)$ and UAF-11 $\times$ BSA had the smallest minimum number of siliquae/secondary branches of plant (800.5). The estimated heterosis effects ranged from 0.507 (Toria $\times$ TP-124-1) to 189.725 (Toria $\times$ UAF-11), and estimated heterobeltiosis effects ranged from -3.50 (Toria $\times$ TP-124-1) to $100.608($ BSA $\times$ Toria $)$. Highly significant positive heterosis was observed in three hybrid progenies: BSA $\times$ Toria, Toria $\times$ UAF-11 and Toria $\times$ BSA. Significant positive heterosis was observed in three hybrid progenies: UAF-11 $\times$ TP-124-1, BSA $\times$ UAF-11 and TP-124-1 $\times$ UAF-11. Non-significant negative heterosis was observed in six hybrid progenies: UAF-11 $\times$ BSA, UAF-11 $\times$ Toria, BSA $\times$ TP-124-1, Toria $\times$ TP-124-1, TP-124-1 $\times$ BSA and TP-124-1 $\times$ Toria. No negative heterosis was observed in the number of siliqua/ secondary branches of plant. Highly significant positive heterobeltiosis was observed in three hybrid progenies: BSA $\times$ Toria, Toria $\times$ UAF-11 and Toria $\times$ BSA. Nonsignificant positive heterobeltiosis was observed in six hybrid progenies: UAF-11 × Toria, UAF-11 × TP-124-1, BSA $\times$ UAF-11, BSA $\times$ TP-124-1, TP-124-1 $\times$ BSA and TP124-1 $\times$ UAF-11. Non-significant negative heterobeltiosis was observed in three hybrid progenies: UAF-11 $\times$ BSA, Toria $\times$ TP-124-1 and TP-124-1 × Toria (Table 4).
Total number of siliquae/plant. Individual mean comparisons of all accessions are shown in the Table 6. Parent Toria had the largest total number siliquae/plant (1261.8) and parent UAF-11 had the lowest total number of siliquae/plant (396.6). Among hybrid progenies, BSA $\times$ Toria had the largest total number of siliquae/plant (2540.5) and UAF-11 $\times$ BSA had the lowest total number of siliquae/ plant (1065.7). The estimated heterosis effects ranged from -3.874 (Toria $\times$ TP-124-1) to $114.276(B S A \times$ Toria), and estimated heterobeltiosis effects ranged from -10.033 $($ Toria $\times$ TP-124-1) to 101.335 (BSA $\times$ Toria). Highly significant positive heterosis was observed in seven hybrid progenies: UAF-11 $\times$ Toria, UAF-11 $\times$ TP-124-1, BSA $\times$ UAF-11, BSA $\times$ Toria, Toria $\times$ UAF-11, Toria $\times$ BSA and TP-124-1 $\times$ UAF-11. Non-significant positive heterosis was observed in three hybrid progenies: UAF-11 $\times$ BSA, BSA $\times$ TP-124-1 and TP-124-1 $\times$ BSA. Non-significant negative heterosis was observed in two hybrid progenies: Toria $\times$ TP-124-1 and TP-124-1 $\times$ Toria. Highly significant positive heterobeltiosis was observed in three hybrid progenies: BSA $\times$ Toria and BSA $\times$ TP-124-1 and TP-124$1 \times$ BSA. Significant positive heterobeltiosis was observed in two hybrid progenies: UAF-11 × TP-124-1 and TP-124$1 \times$ UAF-11. Non-significant positive heterobeltiosis was observed in three hybrid progenies: UAF-11 $\times$ Toria, BSA $\times$ UAF-11 and Toria $\times$ BSA. Highly significant negative heterobeltiosis was observed in one hybrid progeny: Toria $\times$ TP-124-1. Non-significant negative heterobeltiosis was

Table 4. Hybrid vigour of traits related to siliquae number

\begin{tabular}{|c|c|c|c|c|c|c|}
\hline \multirow[t]{2}{*}{ Hybrid progenies } & \multicolumn{2}{|c|}{$\begin{array}{c}\text { No. siliquae / } \\
\text { primary branches of plant }\end{array}$} & \multicolumn{2}{|c|}{$\begin{array}{c}\text { No. siliquae / } \\
\text { secondary branches of plant }\end{array}$} & \multicolumn{2}{|c|}{$\begin{array}{c}\text { Total No. siliquae / } \\
\text { plant }\end{array}$} \\
\hline & heterosis & heterobeltiosis & heterosis & heterobeltiosis & heterosis & heterobeltiosis \\
\hline UAF-11 $\times$ BSA & $6.02 * *$ & $-7.51 \mathrm{~ns}$ & $59.18 \mathrm{~ns}$ & $-2.70 \mathrm{~ns}$ & $41.52 \mathrm{~ns}$ & $-3.94 \mathrm{~ns}$ \\
\hline UAF-11 × Toria & $48.65 * *$ & $25.98 * *$ & $101.99 \mathrm{~ns}$ & $20.37 \mathrm{~ns}$ & $85.24 * *$ & $21.73 \mathrm{~ns}$ \\
\hline UAF-11 × TP-124-1 & $76.51 * *$ & $73.29 * *$ & $111.19^{*}$ & $27.60 \mathrm{~ns}$ & $101.11 * *$ & $36.81^{*}$ \\
\hline $\mathrm{BSA} \times \mathrm{UAF}-11$ & $-1.65 * *$ & $-14.21 \mathrm{~ns}$ & $119.76^{*}$ & $34.33 \mathrm{~ns}$ & $79.43 * *$ & $21.79 \mathrm{~ns}$ \\
\hline BSA $\times$ Toria & $110.63 * *$ & $103.60 * *$ & $115.50 * *$ & $100.61 * *$ & $114.28 * *$ & $101.34 * *$ \\
\hline $\mathrm{BSA} \times \mathrm{TP}-124-1$ & $30.70 * *$ & $15.86^{*}$ & $9.14 \mathrm{~ns}$ & $5.67 \mathrm{~ns}$ & $14.10 \mathrm{~ns}$ & $13.62 * *$ \\
\hline Toria $\times$ UAF-11 & $37.39 * *$ & $16.44^{*}$ & $189.73 * *$ & $72.65 * *$ & $141.90 * *$ & $58.96 * *$ \\
\hline Toria $\times$ BSA & $112.12 * *$ & $105.05^{*}$ & $92.94 * *$ & $79.61 * *$ & $97.75 * *$ & $85.81 \mathrm{~ns}$ \\
\hline Toria $\times$ TP-124-1 & $-19.06^{* *}$ & $-30.34 * *$ & $0.51 \mathrm{~ns}$ & $-3.50 \mathrm{~ns}$ & $-3.87 \mathrm{~ns}$ & $-10.03 * *$ \\
\hline TP-124-1 $\times$ BSA & $24.21 * *$ & $10.10 \mathrm{~ns}$ & $9.51 \mathrm{~ns}$ & $6.03 \mathrm{~ns}$ & $12.89 \mathrm{~ns}$ & $12.42 * *$ \\
\hline TP-124-1 × Toria & $-11.73 * *$ & $-24.02 * *$ & $0.82 \mathrm{~ns}$ & $-3.20 \mathrm{~ns}$ & $-1.99 \mathrm{~ns}$ & $-8.27 \mathrm{~ns}$ \\
\hline TP-124-1 × UAF-11 & $87.08 * *$ & $74.38 * *$ & $114.30 *$ & $29.48 \mathrm{~ns}$ & $106.39 * *$ & $40.40 *$ \\
\hline
\end{tabular}

* - significant, ${ }^{* *}$ - highly significant, ns - non-significant: $\mathrm{t}_{\mathrm{tab}}$ at $15 \mathrm{df}=2.131(\alpha=0.05), 2.947(\alpha=0.01)$

observed in two hybrid progenies: UAF-11 $\times$ BSA and TP-124-1 × Toria (Table 4).

Seed yield/plant (g). Individual mean comparisons of all accessions are shown in Table 6. Parent Toria had the highest seed yield/plant (49.599) and parent UAF-11 had the lowest seed yield/plant (36.635). Among the hybrid progenies, Toria $\times$ UAF-11 had the highest seed yield/plant (92.946) and Toria $\times$ TP-124-1 had the lowest seed yield/plant (31.128). The estimated heterosis effects ranged from $-30.513(\mathrm{TP}-124-1 \times$ Toria) to 115.568 (Toria $\times$ UAF-11), and estimated heterobeltiosis effects ranged from $-37.241(\mathrm{TP}-124-1 \times$ Toria) to 87.396 (Toria $\times$ UAF-11). Highly significant positive heterosis was observed in five hybrid progenies: UAF-11 $\times$ TP-124-1, BSA $\times$ Toria, Toria $\times$ UAF-11, Toria $\times$ BSA and TP-124-1 $\times$ UAF-11. Significant positive heterosis was observed in one hybrid progeny: UAF-11 $\times$ Toria.
Non-significant positive heterosis was observed in one hybrid progeny: BSA $\times$ TP-124-1. Significant negative heterosis was observed in two hybrid progenies: Toria $\times$ TP-124-1 and TP-124-1 $\times$ Toria. Non-significant negative heterosis was observed in three hybrid progenies: UAF$11 \times$ BSA, BSA $\times$ UAF-11 and TP-124-1 × BSA. Highly significant positive heterobeltiosis was observed in five hybrid progenies: UAF-11 $\times$ TP-124-1, BSA $\times$ Toria, Toria $\times$ UAF-11, Toria $\times$ BSA and TP-124-1 $\times$ UAF-11. Non-significant positive heterobeltiosis was observed in one hybrid progeny: UAF-11 $\times$ Toria. Highly significant negative heterobeltiosis was observed in one hybrid progeny: TP-124-1 $\times$ Toria. Significant negative heterosis was observed in one hybrid progeny: Toria $\times$ TP-124-1. Non-significant negative heterobeltiosis was observed in four hybrid progenies: UAF-11 $\times$ BSA, BSA $\times$ UAF-11, $\mathrm{BSA} \times$ TP-124-1 and TP-124-1 $\times$ BSA (Table 5). 
Table 5. Hybrid vigour of seed yield/plant, siliqua length and No. seeds/siliqua

\begin{tabular}{|c|c|c|c|c|c|c|}
\hline \multirow{2}{*}{ Hybrid progenies } & \multicolumn{2}{|c|}{ Seed yield/plant } & \multicolumn{2}{|c|}{ Siliqua length } & \multicolumn{2}{|c|}{ No. seeds/siliqua } \\
\hline & heterosis & heterobeltiosis & heterosis & heterobeltiosis & heterosis & heterobeltiosis \\
\hline UAF-11 $\times$ BSA & $-0.17 \mathrm{~ns}$ & $-8.95 \mathrm{~ns}$ & $11.82 * *$ & $-4.87 * *$ & $-37.28 * *$ & $-60.30 * *$ \\
\hline UAF-11 × Toria & $36.38^{*}$ & $18.56 \mathrm{~ns}$ & $4.69 * *$ & $-5.58 * *$ & $-38.05 * *$ & $-59.44 * *$ \\
\hline UAF-11 × TP-124-1 & $85.03 * *$ & $77.27 * *$ & $-7.91 * *$ & $-12.06^{* *}$ & $-27.80 * *$ & $-54.57 * *$ \\
\hline $\mathrm{BSA} \times \mathrm{UAF}-11$ & $-3.86 \mathrm{~ns}$ & $-12.31 \mathrm{~ns}$ & $3.24 *$ & $-12.17 * *$ & $-37.17 * *$ & $-60.23 * *$ \\
\hline BSA $\times$ Toria & $93.76^{* *}$ & $83.72 * *$ & $5.04 * *$ & $-1.63 * *$ & $51.92 * *$ & $41.20 * *$ \\
\hline $\mathrm{BSA} \times \mathrm{TP}-124-1$ & $3.28 \mathrm{~ns}$ & $-1.90 \mathrm{~ns}$ & $-1.16 \mathrm{~ns}$ & $-19.03 * *$ & $18.87 * *$ & $17.19 * *$ \\
\hline Toria $\times$ UAF-11 & $115.57 * *$ & $87.40 * *$ & $-11.45 * *$ & $-20.14 * *$ & $-39.37 * *$ & $-60.30 * *$ \\
\hline Toria $\times$ BSA & $47.73 * *$ & $40.07 * *$ & $8.27^{* *}$ & $1.39 \mathrm{~ns}$ & $51.72 * *$ & $41.03 * *$ \\
\hline Toria $\times$ TP-124-1 & $-28.18^{*}$ & $-35.13 *$ & $1.04 \mathrm{~ns}$ & $-12.54 * *$ & $14.91 * *$ & $5.41 \mathrm{~ns}$ \\
\hline TP-124-1 × BSA & $-3.03 \mathrm{~ns}$ & $-7.90 \mathrm{~ns}$ & $-0.22 \mathrm{~ns}$ & $-18.26^{* *}$ & $18.31 * *$ & $16.64 * *$ \\
\hline TP-124-1 × Toria & $-30.51 *$ & $-37.24 * *$ & $1.44 \mathrm{~ns}$ & $-12.19 * *$ & $15.99 * *$ & $6.41 * *$ \\
\hline TP-124-1 × UAF-11 & $82.65 * *$ & $74.98 * *$ & $-7.69 \mathrm{~ns}$ & $-11.84 * *$ & $-24.99 * *$ & $-52.80 * *$ \\
\hline
\end{tabular}

* - significant, $* *$ - highly significant, $\mathrm{ns}$ - non-significant: $\mathrm{t}_{\text {tab }}$ at $15 \mathrm{df}=2.131(\alpha=0.05), 2.947(\alpha=0.01)$

Siliqua length (cm). Individual mean comparisons of all accessions are shown in the Table 6. Parent TP-124-1 had the longest siliqua length (6.91) and parent BSA had the shortest siliqua length (4.41). Among the hybrid progenies, TP-124-1 $\times$ UAF-11 had the longest siliqua length (6.09) and BSA $\times$ Toria had the shortest siliqua length (4.97). The estimated heterosis effects ranged from -11.448 (Toria $\times$ UAF-11) to 11.818 $(\mathrm{UAF}-11 \times \mathrm{BSA})$, and estimated heterobeltiosis effects ranged from -20.140 (Toria $\times$ UAF-11) to 1.386 (Toria $\times$ BSA). Highly significant positive heterosis was observed in four hybrid progenies: UAF-11 $\times$ BSA, UAF-11 $\times$ Toria, BSA $\times$ Toria and Toria $\times$ BSA. Significant positive heterosis was observed in one hybrid progeny: BSA $\times$ UAF-11. Non-significant positive heterosis was observed in two hybrid progenies: Toria $\times$ TP-124-1 and TP-124-1 $\times$ Toria. Highly significant negative heterosis was observed in two hybrid progenies: UAF-11 $\times \mathrm{TP}$ 124-1 and Toria $\times$ UAF-11. Non-significant negative heterosis was observed in three hybrid progenies: BSA $\times$ TP-124-1, TP-124-1 $\times$ BSA and TP-124-1 $\times$ UAF11. No significant positive heterobeltiosis was seen.
Non-significant positive heterobeltiosis was observed in one hybrid progeny: Toria $\times$ BSA. Highly significant negative heterobeltiosis was observed in all other hybrid progenies (Table 5).

Number of seeds/siliqua. Individual mean comparisons of all accessions are shown in Table 6 . Parent UAF-11 had the greatest number of seeds/siliqua (45.34) and parent BSA had the lowest number of seeds/ siliqua (12.06). Among the hybrid progenies, TP-124-1 $\times$ UAF-11 had the greatest number of seeds/siliqua (21.4) and TP-124-1 $\times$ BSA had the lowest number of seeds/ siliqua (14.067). The estimated heterosis effects ranged from -39.374 (Toria $\times$ UAF-11) to $51.916(\mathrm{BSA} \times$ Toria), and estimated heterobeltiosis effects ranged from -60.300 (Toria $\times$ UAF-11, UAF-11 $\times$ BSA) to 41.204 $(\mathrm{BSA} \times$ Toria). Highly significant positive heterosis was observed in six hybrid progenies: BSA $\times$ Toria, BSA $\times$ TP-124-1, Toria $\times$ BSA, Toria $\times$ TP-124-1, TP-124-1 $\times$ BSA and TP-124-1 $\times$ Toria. No non-significant positive heterosis was observed in the number of seeds/siliqua. Highly significant negative heterosis was observed in six hybrid progenies: UAF-11 × BSA, UAF-11 × Toria,

Table 6. Mean performance of parents and hybrid progenies for seed yield-related traits

\begin{tabular}{cccccccccc}
\hline Parents $/$ crosses & SV & PH & $\begin{array}{c}1000 \mathrm{seed} \\
\text { weight }\end{array}$ & NSPB & NSSB & TNSP & SYP & SL & NSS \\
\hline BSA & $5.58 \mathrm{~K}$ & $174.9 \mathrm{~A}$ & $2.39 \mathrm{HI}$ & $286.70 \mathrm{FG}$ & $822.7 \mathrm{CD}$ & $1109.4 \mathrm{D}$ & $44.46 \mathrm{~F}$ & $4.41 \mathrm{H}$ & $12.06 \mathrm{I}$ \\
BSA $\times$ UAF-11 & $5.35 \mathrm{~L}$ & $128.2 \mathrm{DEFG}$ & $2.30 \mathrm{IJ}$ & $245.97 \mathrm{GHI}$ & $1105.2 \mathrm{BCD}$ & $1351.1 \mathrm{CD}$ & $38.98 \mathrm{H}$ & $5.52 \mathrm{E}$ & $18.03 \mathrm{E}$ \\
BSA $\times$ Toria & $5.78 \mathrm{~J}$ & $128.9 \mathrm{CDEF}$ & $2.43 \mathrm{H}$ & $625.45 \mathrm{~A}$ & $1915.0 \mathrm{~A}$ & $2540.5 \mathrm{~A}$ & $91.12 \mathrm{~B}$ & $4.97 \mathrm{G}$ & $19.83 \mathrm{D}$ \\
BSA $\times$ TP-124-1 & $7.45 \mathrm{BC}$ & $127.6 \mathrm{DEFG}$ & $4.78 \mathrm{~A}$ & $332.17 \mathrm{DE}$ & $928.3 \mathrm{BCD}$ & $1260.5 \mathrm{CD}$ & $43.61 \mathrm{~F}$ & $5.59 \mathrm{E}$ & $14.13 \mathrm{GH}$ \\
Toria & $6.39 \mathrm{H}$ & $145.2 \mathrm{~B}$ & $2.97 \mathrm{G}$ & $307.20 \mathrm{EF}$ & $954.6 \mathrm{BCD}$ & $1261.8 \mathrm{CD}$ & $49.60 \mathrm{E}$ & $5.05 \mathrm{FG}$ & $14.04 \mathrm{H}$ \\
Toria $\times$ UAF-11 & $7.31 \mathrm{DE}$ & $123.2 \mathrm{EFGH}$ & $3.31 \mathrm{E}$ & $357.70 \mathrm{CD}$ & $1648.1 \mathrm{~A}$ & $2005.8 \mathrm{~B}$ & $92.95 \mathrm{~A}$ & $5.02 \mathrm{FG}$ & $18.00 \mathrm{E}$ \\
Toria $\times$ BSA & $5.97 \mathrm{I}$ & $122.2 \mathrm{FGH}$ & $3.11 \mathrm{~F}$ & $629.90 \mathrm{~A}$ & $1714.6 \mathrm{~A}$ & $2344.5 \mathrm{AB}$ & $69.47 \mathrm{C}$ & $5.12 \mathrm{~F}$ & $19.80 \mathrm{D}$ \\
Toria $\times$ TP-124-1 & $6.77 \mathrm{G}$ & $131.2 \mathrm{CDE}$ & $3.97 \mathrm{D}$ & $214.00 \mathrm{I}$ & $921.2 \mathrm{BCD}$ & $1135.2 \mathrm{D}$ & $32.17 \mathrm{~J}$ & $6.04 \mathrm{CD}$ & $14.80 \mathrm{FG}$ \\
TP-124-1 & $7.86 \mathrm{~A}$ & $120.4 \mathrm{GH}$ & $4.39 \mathrm{~B}$ & $221.60 \mathrm{I}$ & $878.5 \mathrm{BCD}$ & $1100.1 \mathrm{D}$ & $39.99 \mathrm{GH}$ & $6.91 \mathrm{~A}$ & $11.72 \mathrm{I}$ \\
TP-124-1 $\times$ UAF-11 & $6.85 \mathrm{FG}$ & $121.5 \mathrm{FGH}$ & $4.20 \mathrm{C}$ & $407.00 \mathrm{~B}$ & $1137.5 \mathrm{BC}$ & $1544.5 \mathrm{C}$ & $69.98 \mathrm{C}$ & $6.09 \mathrm{C}$ & $21.40 \mathrm{~B}$ \\
TP-124-1 $\times$ BSA & $7.51 \mathrm{~B}$ & $136.8 \mathrm{C}$ & $4.80 \mathrm{~A}$ & $315.67 \mathrm{EF}$ & $931.5 \mathrm{BCD}$ & $1247.2 \mathrm{CD}$ & $40.95 \mathrm{G}$ & $5.65 \mathrm{E}$ & $14.07 \mathrm{GH}$ \\
TP-124-1 $\times$ Toria & $6.80 \mathrm{G}$ & $132.4 \mathrm{CD}$ & $4.43 \mathrm{~B}$ & $233.40 \mathrm{HI}$ & $924.1 \mathrm{BCD}$ & $1157.5 \mathrm{D}$ & $31.12 \mathrm{~J}$ & $6.067 \mathrm{CD}$ & $14.94 \mathrm{~F}$ \\
UAF-11 & $7.20 \mathrm{E}$ & $93.7 \mathrm{I}$ & $3.91 \mathrm{D}$ & $213.50 \mathrm{I}$ & $183.1 \mathrm{E}$ & $396.6 \mathrm{E}$ & $36.64 \mathrm{I}$ & $6.28 \mathrm{~B}$ & $45.34 \mathrm{~A}$ \\
UAF-11 $\times$ BSA & $5.51 \mathrm{~K}$ & $123.1 \mathrm{EFGH}$ & $2.19 \mathrm{~J}$ & $265.17 \mathrm{GH}$ & $800.5 \mathrm{D}$ & $1065.7 \mathrm{D}$ & $40.48 \mathrm{GH}$ & $5.98 \mathrm{CD}$ & $18.00 \mathrm{E}$ \\
UAF-11 $\times$ Toria & $7.37 \mathrm{CD}$ & $128.0 \mathrm{DEFG}$ & $3.41 \mathrm{E}$ & $387.00 \mathrm{BC}$ & $1149.0 \mathrm{~B}$ & $1536.0 \mathrm{C}$ & $58.80 \mathrm{D}$ & $5.94 \mathrm{D}$ & $18.40 \mathrm{E}$ \\
UAF-11 $\times$ TP-124-1 & $6.92 \mathrm{~F}$ & $116.7 \mathrm{H}$ & $4.25 \mathrm{C}$ & $384.00 \mathrm{BC}$ & $1121.0 \mathrm{BCD}$ & $1505.0 \mathrm{C}$ & $70.90 \mathrm{C}$ & $6.07 \mathrm{CD}$ & $20.60 \mathrm{C}$ \\
\hline
\end{tabular}

Note. SV - seed volume, PH - plant height, NSPB - number of siliquae/primary branches of plant, NSSB - number of siliquae/ secondary branches of plant, TNSP - total number of siliquae/plant, SYP - seed yield/plant, SL - siliqua length, NSS - number of seeds/siliqua; superscript capital letters indicate similarities between different genotypes. 
UAF-11 $\times$ TP-124-1, BSA $\times$ UAF-11, Toria $\times$ UAF-11 and TP-124-1 $\times$ UAF-11. No non-significant negative heterosis was observed for the number of seeds/siliqua. Highly significant positive heterobeltiosis was observed in five hybrid progenies: BSA $\times$ Toria, BSA $\times$ TP-124-1, Toria $\times$ BSA, TP-124-1 $\times$ BSA and TP-124-1 $\times$ Toria. Non-significant positive heterobeltiosis was observed in one hybrid: Toria $\times$ TP124-1. Highly significant negative heterobeltiosis was observed in six hybrid progenies: UAF-11 × BSA, UAF-11 × Toria, UAF-11 × TP-124-1, BSA $\times$ UAF-11, Toria $\times$ UAF-11 and TP-124-1 $\times$ UAF11 (Table 5).

Correlation. Correlation coefficient is helpful in selection of better plants. Correlation helps in determining how strongly the pairs of traits are related. The relationship between yield and various morphological characters of the plant population which influence yield are very great indeed, as it furnishes the plant breeder with an easy and fairly reliable means of isolating high yielding and better quality genotypes from the breeding material (Khan et al., 2008). In this study, significant positive correlation was found for seed volume with 1000 seed weight and siliqua length, 1000 seed weight and silliqua length, number of seeds/primary branches of plant with number of seeds/secondary branches of plant, total number of seeds/plant and seed yield/plant, number of seeds/secondary branches of plant with total number of seeds/plant and seed yield/plant, total number of seeds/ plant and seed yield per plant. A negative correlation was found for plant height with siliqua length and number of seeds/siliqua (Table 7).

Table 7. Correlation of seed yield-related traits

\begin{tabular}{cccccccccc}
\hline Variables & SV & PH & 1000 SW & NSPB & NSSB & TNSP & SYP & SL & NSS \\
\hline SV & $\mathbf{1}$ & -0.370 & $\mathbf{0 . 8 5 5}$ & -0.239 & -0.231 & -0.241 & -0.048 & $\mathbf{0 . 5 4 8}$ & 0.066 \\
PH & -0.370 & $\mathbf{1}$ & -0.313 & -0.031 & 0.104 & 0.074 & -0.116 & $\mathbf{- 0 . 6 4 5}$ & $\mathbf{- 0 . 7 0 4}$ \\
1000SW & $\mathbf{0 . 8 5 5}$ & -0.313 & $\mathbf{1}$ & -0.236 & -0.293 & -0.289 & -0.227 & $\mathbf{0 . 5 7 5}$ & 0.011 \\
NSPB & -0.239 & -0.031 & -0.236 & $\mathbf{1}$ & $\mathbf{0 . 8 2 4}$ & $\mathbf{0 . 8 9 5}$ & $\mathbf{0 . 7 8 3}$ & -0.448 & 0.000 \\
NSSB & -0.231 & 0.104 & -0.293 & $\mathbf{0 . 8 2 4}$ & $\mathbf{1}$ & $\mathbf{0 . 9 9 0}$ & $\mathbf{0 . 8 1 4}$ & -0.474 & -0.334 \\
TNSP & -0.241 & 0.074 & -0.289 & $\mathbf{0 . 8 9 5}$ & $\mathbf{0 . 9 9 0}$ & $\mathbf{1}$ & $\mathbf{0 . 8 3 3}$ & -0.483 & -0.262 \\
SYP & -0.048 & -0.116 & -0.227 & $\mathbf{0 . 7 8 3}$ & $\mathbf{0 . 8 1 4}$ & $\mathbf{0 . 8 3 3}$ & $\mathbf{1}$ & -0.405 & 0.051 \\
SL & $\mathbf{0 . 5 4 8}$ & $\mathbf{- 0 . 6 4 5}$ & $\mathbf{0 . 5 7 5}$ & -0.448 & -0.474 & -0.483 & -0.405 & $\mathbf{1}$ & 0.233 \\
NSS & 0.066 & $\mathbf{- 0 . 7 0 4}$ & 0.011 & 0.000 & -0.334 & -0.262 & 0.051 & 0.233 & $\mathbf{1}$ \\
\hline
\end{tabular}

Note. SV - seed volume, PH - plant height, 1000SW - 1000 seed weight, NSPB - number of seeds/primary branches of plant, NSSB - number of seeds/secondary branches of plant, TNSP - total number of seeds/plant, SYP - seed yield/plant, SL - siliqua length, NSS - number of seeds/siliqua, significant correlations are in bold.

\section{Discussion}

Seed colour variations in B. rapa is common, with brown seeds ranging from dark brown to reddish brown and yellow seeds ranging from yellow-brown to yellow. The most common colour is brown, but yellow seed is desirable due to its higher oil content, lower crude fibre content, and protein content in meal (Jonsson, Bengtsson, 1970; Stringam et al., 1974). Ahmad and Zuberi (1971) reported that seed colour in B. rapa is controlled by a single gene, with brown being dominant over yellow. Schwetka (1982) reported that seed colour in $B$. rapa is controlled by one or two genes with epistatic effects. He also reported maternal parent influence to some extent in seed colour determination. Stringam (1980) reported digenic control of seed colour in B. rapa by two independent genes, $\mathrm{Br}_{1}$ and $\mathrm{Br}_{3}$, while Mohammad et al. (1942) and Jonsson (1975) reported that seed colour in $B$. rapa is controlled by three genes which segregate independently. According to these authors, brown seed colour is dominant over yellow, which appears only when all loci are homozygous recessive. The results of the present study support digenic control of this seed trait, given that the results of reciprocal crosses indicated that seed colour is a pure heritable trait that is little affected by the environment. Genetic control of seed colour indicated that this trait is controlled by a major gene. Seed colour showed complete Mendelian genetics as clearly seen in the Punnett table (Table 2). Our findings further show that seed colour is not influenced by maternal effects.

A single seed shape shared by both parents and all hybrid progenies in the present study indicates that there was little variation in seed shape across $B$. rapa accessions. Our results strongly suggest that seed shape is controlled by a (probably single) major gene.

Leaf hairiness plays an important role in protecting the plant from abiotic stresses such as ultraviolet radiation and drought, and from biotic stresses such as disease and insect infestation (Skaltsa et al., 1994). Rahman (2014) reported that leaf hairiness was controlled by a single gene, and that hairiness was dominant over non-hairiness. The results of the present research also support this conclusion.

Leaf shape variations are frequent in the Brassica group and lanceolate, spatulate, obovate, broad-elliptic, broad-circular and pandurate shapes are seen most commonly in this group (Zada et al., 2013). Leaf shape is under digenic control, and it was clear from our results that genes for leaf shapes have multiple alleles. Variations in leaf colour occur in the Brassica group although the most frequent colours are generally light green, dark green, yellow green and purple (Zada et al., 2013). Our results strongly suggest that leaf colour is under digenic control, with green being the dominant colour overall, and darker colours also dominating.

Ahmed (2010) observed that better and midparent heterosis was significant for most traits, and reported the highest heterosis for seed yield. He concluded that heterosis breeding is an economical and feasible method for the production of commercial hybrid progenies of B. rapa. Sabaghnia et al. (2010) estimated heterosis in nine genotypes from five countries, e.g., Germany, Denmark, Iran, France and Sweden, crossed in a complete diallel mating design. They observed significant positive mid-parent and high parent heterosis for all traits in different crosses, and concluded that $F_{1}$ hybrid progenies 
Zarfam $\times$ Orient, Talaye $\times$ Orient and Talaye $\times$ Opera performed best in terms of heterosis. They recommended these genotypes for use as a source population for further selection or as $\mathrm{F}_{1}$ hybrid progenies. Dar et al. (2012) estimated heterosis for seed yield-related traits in brown Brassica (B. rapa) in ten different genotypes: KOS101, KOS-1, CR-1617, CR-2638, CR-2871, CR-2677, CR-1480, CR-1485, CR-1607 and CR-1630. They found that CR-1485 $\times$ CR-1607 was the most desirable cross combination for seed yield and also estimated desirable better and mid-parent heterosis for primary branches of the plant and 1000 seed weight. They observed the highest mid-parent and better parent heterosis in KOS-1 $\times$ CR-2638 for number of siliquae on the main raceme, CR-1607 $\times$ CR-1485 for primary branches of plant, CRKOS-101 $\times$ CR-1630 for secondary branches of plant, KOS- $1 \times$ CR-1607 for days to maturity and CR-2871 $\times$ CR-1630 for number of siliquae/plant.

We found significant negative heterosis for plant height in BSA $\times$ Toria, BSA $\times$ TP-124-1 and Toria $\times$ BSA crosses, and the highest heterosis and heterobeltiosis for seed volume in UAF-11 $\times$ Toria. The highest heterosis and heterobeltiosis (significantly higher than in other crosses) for 1000 seed weight were found in BSA $\times$ TP-124-1, Toria $\times$ BSA and TP-124, the and the highest heterosis and heterobeltiosis for number of siliquae/primary braches (significantly higher than in other crosses) were seen in UAF-11 $\times$ Toria, UAF$11 \times$ TP-124-1, BSA $\times$ Toria and TP-124-1 $\times$ UAF-11. The highest heterosis and heterobeltiosis (significantly higher than in other crosses) for number of siliquae/ secondary branches of plant were found in BSA $\times$ Toria, Toria $\times$ UAF-11 and Toria $\times$ BSA, and highest heterosis and heterobeltiosis (significantly higher than in other crosses) for total number of siliquae/plant were seen in BSA $\times$ Toria and Toria $\times$ UAF-11 crosses. The highest heterosis and heterobeltiosis (significantly higher than in other crosses) for seed yield/plant were seen in UAF$11 \times$ TP-124-1, BSA $\times$ Toria, Toria $\times$ UAF-11, Toria $\times$ BSA and TP-124-1 $\times$ UAF-11.The highest heterosis and heterobeltiosis (significantly higher than in other crosses) for siliqua length were found in UAF- $11 \times \mathrm{BSA}$, UAF$11 \times$ Toria, BSA $\times$ Toria and Toria $\times$ BSA. The highest heterosis and heterobeltiosis (significantly higher than in other crosses) for number of seeds/silique were seen in BSA $\times$ Toria, BSA $\times$ TP-124-1, Toria $\times$ BSA, TP-124-1 $\times$ BSA and TP-124-1 $\times$ Toria. UAF-11 and TP-124-1 can be used as parents in future hybrid breeding programs after evaluating their combing ability effects.

In correlation analysis seed yield was significantly correlated with number of seeds/primary branches of plant, number of seeds/secondary branches of plant and total number of seeds/plant, while number of seeds/primary branches of plant and number of seeds/ secondary branches of plant both were significantly correlated with total number of seeds/plant, these results clearly indicated that these were most important traits for seed yield/plant while negative correlation of plant height indicated that short height was desirable for increasing seed yield. Except plant height all others had positive correlation. From these results it can be inferred that for increasing seed yield selection would be very effective for number of seeds/primary branches of plant and number of seeds/secondary branches of plant in positive manner and for plant height in negative manner. Khayat et al. (2012) found positive and significant correlation between 1000 seed weight and seed yield. Keeping in consideration Khayat et al. (2012) findings significant and positive correlation of 1000 seed weight with seed volume and siliqua length indicated that improvement in seed volume and siliqua length would be helpful in increasing seed yield. Malik et al. (2000) also observed positive correlation of seed yield with siliqua length, number of seeds/primary branches of plant and number of seeds/secondary branches of plant. They also found negative and non-significant correlation for seed yield with 1000 seed weight and seed/siliqua which is contrary to these findings. Ali et al. (2013) also reported similar findings, they found positive and significant correlation between seed weight and seed yield. Our results for the traits, compared here will, hopefully, facilitate future efforts to select productive $B$. rapa accessions for breeding programs aimed at optimizing heterosis. Our results are consistent with early findings published by Oghan et al. (2007), Pankaj et al. (2008), Ahmed (2010), Sabaghnia et al. (2010), Dar et al. (2012), Rameeh (2012), Ahsan et al. (2013), Ali et al. (2013), Nasim et al. (2013), Zada et al. (2013), Muhammad et al. (2014) and Mumtaz et al. (2016).

\section{Conclusion}

For efficient use of oilseed rape (Brasssica rapa L.) in overcoming the gap between production and consumption of edible oil, genetics of $B$. rapa should be known. In order to ascertain genetics of qualitative (seed colour, seed shape, leaf hairiness, leaf shape and leaf colour) and seed yield-related traits (plant height, seed volume, 1000 seed weight, number of siliquae/ primary branches of plant, number of siliquae/secondary branches of plant, number of siliquae/total branches of plant, siliqua length, number of seeds/siliqua and seed yield/ plant) of B. rapa, study of Mendelian genetics and heterosis revealed that seed colour behaved according to Mendelian genetics in digenic inheritance pattern, appearance of leaf hairiness in all crosses including self, indicated its monogenic inheritance. Leaf shape pandurate was dominant over all other shapes and elliptic was recessive to all. Leaf colour showed large variation, green was dominant over all. Number of siliquae/primary branches of plant, number of siliquae/secondary branches of plant and total number of siliquae/plant directly affect seed yield of plant while effect of plant height is indirect. In addition, 1000 seed weight, seed volume and number of seeds/siliqua also contributed to seed yield/plant. For increasing seed yield, selection would be very effective for number of siliquae/primary branches of plant and number of siliquae/secondary branches of plant in positive manner and plant height in negative manner. TP124-1 × UAF-11 and its reciprocal cross heterosis and herobeltiosis was best for all traits except plant height. From this, it can be suggested that TP-124-1 and UAF11 can be used in hybrid breeding program as parents after evaluating their combining abilities. No variation was observed in seed shape but further testing for seed shape is required. 


\section{Acknowledgements}

The first author is grateful to Dr. H. A. Sadaqat for his guidance during my research, and to the Oilseed Research Institute, Ayub Agriculture Research Institute, Faisalabad, Pakistan for providing genetic material. I express appreciation to the staff of the sunflower laboratory for their help and encouragement, and to K. Shashok (AuthorAID in the Eastern Mediterranean) for editing the manuscript. I dedicate this article to my parents, teachers, sisters, brothers and friends for their prayers and encouragement.

Received 02092016 Accepted 15022017

\section{References}

Ahmad S. U., Zuberi M. I. 1971. Inheritance of seed coat color in Brassica campestris L. variety Toria. Crop Science, 11 (2): 309-310 https://doi.org/10.2135/cropsci1971.001118 3X001100020047x

Ahmed Z. 2010. Genetic studies in brown sarson. PhD thesis, Department of Plant Breeding and Genetics, University of Agricultural Sciences Srinagar, India

Ahsan M. Z., Khan F. A., Kang S. A., Rasheed K. 2013. Combining ability and heterosis analysis for seed yield and yield components in Brassica napus L. Journal of Biology, Agriculture and Healthcare, 3: 2224-3208

Ali Y., Farhatullah, Rahman H., Nasim A., Azam S. M., Khan A. 2013. Heritability and correlation analysis for morphological and biochemical traits in Brassica carinata. Sarhad Journal of Agriculture, 29 (3): 359-370

Dar Z. A., Wani S. A., Gulzaffar, Habib M., Sofi N. R., Ahmed I., Ahmed Z., Khan M. H. 2012. Heterosis studies in brown sarson (Brassica rapa L.). Electronic Journal of Plant Breeding, 3 (1): 676-681

Falconer D. S., Mackay T. F. C. 1996. Introduction to quantitative genetics $\left(4^{\text {th }}\right.$ ed.). New York, USA

Government of Pakistan. 2015-2016. Ministry of Finance, Economic Advisor' Wing, Islamabad

Jonsson R. 1975. Yellow-seeded rape and turnip rape. II Breeding for improved quality of oil and meal in yellowseeded materials. Sveriges Utsadesforenings Tidsk rift, 85: 271-278 (in Swedish)

Jonsson R., Bengtsson L. 1970. Yellow-seeded rape and turnip rape. 1. Influence of breeding for yellow seeds upon yield and quality properties. Sveriges Utsadesforenings Tidsk rift, 80: 149-155

Khan S., Farhatullah, Khalil I. H., Munir I., Khan M. Y., Ali N. 2008. Genetic variability for morphological traits in $F_{3}$ Brassica populations. Sarhad Journal of Agriculture, 24 (2): 217-222

Kumar H., Anubha, Vishwakarma M. K., Lal J. P. 2011. Morphological and molecular characterization of Brassica rapa ssp. yellow sarson mutants. Journal of Oilseed Brassica, 2 (1): 1-6

Khayat M., Lack S. H., Karami H. 2012. Correlation and path analysis of traits affecting grain yield of canola (Brassica napus L.) varieties. Journal of Basic and Applied Scientific Research, 2 (6): 5555-5562

Malik M. A., Khan A. S., Shafiullah, Ayub M. K. 2000. Study of correlation among morphological parameters in different varietites/accessions of Brassica species. Pakistan Journal of Biology and Science, 3 (7): 1180-1182 https://doi.org/10.3923/pjbs.2000.1180.1182

Mead R., Curnow R. N., Hasted A. M. 2002. Statistical methods in agriculture and experimental biology ( $3^{\text {rd }}$ ed.). Boca Raton, USA, 488 p.
Mohammad A., Sikka S. M., Aziz M. A. 1942. Inheritance of seed color in some oliferous Brassica. Indian Journal of Genetics and Plant Breeding, 2: 112-127

Muhammad A., Raziuddin, Muhammad A., Raza H., Rahman A., Ali I. 2014. Combining ability and heritability studies for important traits in $\mathrm{F}_{2}$ populations of Brassica napus L. International Journal of Basic and Applied Sciences, 14 (01): 7-11

Mumtaz A., Sadaqat H. A., Saifulmalook, Nazik A. S., Ahmad H. M. 2014. Genetic behaviour of quality related traits in Brassica rapa. Vegetos, 27 (3): 139-145 https://doi.org/10.5958/2229-4473.2014.00081.0

Mumtaz A., Sadaqat H. A., Mubarik M. K., Malook S., Nawaz M. A., Asif M., Hussain W. 2015. A review on genetics of seed yield and quality related traits in Brassica group. American-Eurasian Journal of Agriculture and Environmental Sciences, 15 (7): 1213-1220

Mumtaz A., Sadaqat H. A., Yousaf M. I., Saeed M., Zaman R. Q., Shehzad A., Rana H. S. 2016. Gene action studies in Brassica rapa for seed yield related traits. Journal of Global Innovation in Agriculture and Social Sciences, 4 (4): $160-166$ https://doi.org/10.22194/JGIASS/4.4.764

Nasim A., Farhatullah, Iqbal S., Shah S., Azam S. M. 2013. Genetic variability and correlation studies for morphophysiological traits in Brassica napus L. Pakistan Journal of Botany, 45: 1229-1234

Oghan A., Shariati H., Asadi F., Tusi M., Kamyab F., Fatemeh. 2007. Study of heterosis and combining ability of yield and yield components in spring rapeseed. Food and Agriculture Organization of United Nations $<\mathrm{http}$ ://agris.fao.org/agrissearch/search.do?recordID=IR2008000483>

Pankaj S., Pant D. P., Singh S. P., Singh A. K., Rakesh S. 2008. Diallel analysis for yield and yield component traits in Toria (Brassica rapa L.). Indian Journal of Plant Genetic Resources, 21: 121-124

Rahman M. 2014. Independent assortment of seed color and leaf hairiness genes in Brassica rapa L. Canadian Journal of Plant Sciences, 94 (4): 615-620 https://doi.org/10.4141/cjps2012-323

Rahman M., McVetty P. B. E. 2011. A review of Brassica seed color. Canadian Journal of Plant Science, 91: 437-446 https://doi.org/10.4141/cjps10124

Rameeh V. 2012. Combining ability analysis of plant height and yield components in spring type of rapeseed varieties (Brassica napus $\mathrm{L}$.) using line $\times$ tester analysis. International Journal of Agriculture and Forestry, 2: 58-62 https://doi.org/10.5923/j.ijaf.20120201.10

Rather A. G., Sheikh F. A., Najeeb S., Zargar M. A., Parray G. A., Shikari A. B., Ahangar M. A., Bhat Z. A., Ahmed S. 2012. Development of yellow seeded brown sarson (B. rapa L.) genotypes for temperate conditions of Kashmir. Electronic Journal of Plant Breeding, 3: 1018-1020

Sabaghnia N., Dehghani H., Alizadeh B., Mohghaddam M. 2010. Heterosis and combining ability analysis for oil yield and its components in rapeseed. Australian Journal of Crop Science, 4: 390-397

Schwetka A. 1982. Inheritance of seed color in turnip rape (Brassica campestris L.). Theoretical and Applied Genetics, 62: 161-169 https://doi.org/10.1007/BF00293352

Sinhamahapatra S. P., Raman R. B., Roy S., Roy U. S., Raut N. M., Ashok K. V. 2010. Breeding for an ideal plant type in yellow sarson (Brassica rapa L. yellow sarson). Electronics Journal of Plant Breeding, 1: 689-694

Skaltsa H., Verykokidou E., Harvala C., Karabourniotis G., Manetas Y. 1994. UV-B protective potential and flavonoid content of leaf hairs of quercusilex. Phytochemistry, 37: 987-990

https://doi.org/10.1016/S0031-9422(00)89514-X 
Stringam G. R. 1980. Inheritance of seed color in turnip rape. Canadian Journal of Plant Science, 60 (2): 331-335 https://doi.org/10.4141/cjps80-054

Stringam G. R., McGregor D. I., Pawlowski S. H. 1974. Chemical and morphological characteristics associated with seed-coat color in rapeseed. Proceedings of the $4^{\text {th }}$ International Rapeseed Conference. Giessen, Germany, p. $99-108$

Vera C. L., Woods D. L., Downey R. K. 1979. Inheritance of seed coat color in B. juncea. Canadian Journal Plant Science, 59: 635-637

https://doi.org/10.4141/cjps79-100
Zada M., Zakir N., Rabbani M. A., Shinwari Z. K. 2013. Assessment of genetic variation in Ethiopian mustard (Brassica carinata Braun) germplasm using multivariate techniques. Pakistan Journal of Botany, 45: 583-593

Zhang Y., Li X., Ma C., Chen J., Chen B., Tu J., Fu T. 2009. The inheritance of seed color in a resynthesized Brassica napus line No. 2127-17 including a new epistatic locus. Genes Genomics, 31: 413-419 https://doi.org/10.1007/BF03191854

ISSN 1392-3196 / e-ISSN 2335-8947

Zemdirbyste-Agriculture, vol. 104, No. 2 (2017), p. 147-156

DOI 10.13080/z-a.2017.104.019

\title{
Aliejinio rapso kokybiniai ir su sèklų derliumi susiję genetiniai požymiai
}

\author{
A. Mumtaz ${ }^{1,2}$, H. A. Sadaqat², M. Saeed ${ }^{1}$, M. I. Yousaf ${ }^{1,2}$, A. Shehzad'2, H. G. M. D. Ahmed ${ }^{2}$ \\ ${ }^{1}$ Kukurūzų ir sorų tyrimo institutas, Pakistanas \\ ${ }^{2}$ Pakistano žemès ūkio universitetas
}

\section{Santrauka}

Maistinio aliejaus, pagaminto vidaus rinkoje, kiekis tenkina tik 20,23\% Pakistano paklausos, todèl reikia didinti jo gamybą. Dėl trumpo vegetacijos laikotarpio, didelio sẻklų derliaus ir geltonos spalvos sèklų aliejinis rapsas (Brassica rapa L.) yra potencialiai naudingas augalas, galintis užpildyti atotrūkị tarp gamybos ir vartojimo. Tyrimo tikslas - nustatyti genetinę aprašytų ir su sėklų derliumi susijusių požymių raišką keturiose aliejinio rapso linijose (UAF-11, Toria, BSA ir TP-124-1) ir jų hibridiniuose palikuonyse, gautuose taikant pilną dialelinio kryžminimo schemą; taip pat ivertinta heterozè ir heterobeltiozè. Sẻklos buvo ịvairiu atspalviu rudos spalvos ir pasiskirstė pagal Mendelio dèsnius. Visų linijų sẻklos buvo apvalios. Lapų plaukuotumas buvo būdingas visų kryžminimo kombinacijų palikuonims, taip pat ir savidulkiams augalams. Visos tèvinės linijos turejjo savitą lapų formą elipsinę, smuikišką arba ietišką. Tarpinių ar naujų lapų formų $F_{1}$ kartos palikuonyse nebuvo nustatyta. Lapai buvo įvairių žalios spalvos atspalvių. Sẻklų bei lapų formą ir lapų spalvą kontroliavo du genai, epistatinio ar motininio poveikio nebuvo nustatyta. Lapų formai buvo būdingas daugybinis alelizmas. Sèklų forma variacijų neturèjo. Lapų plaukuotumas buvo dominuojantis ir monogeniškai kontroliuojamas požymis. Tiesioginès įtakos sẻklų derliui turejjo ankštarų bei pagrindinių šakų ir ankštarų bei šalutinių šakų skaičius augale ir bendras ankštarų skaičius augale, o augalų aukščio įtaka buvo netiesioginè. Visiems požymiams nustatyta heterozès ir heterobeltiozès variacija. Linija UAF-11 ir jos kryžminimo kombinacijos su Toria bei TP-124-1 ir jų ekvivalentai buvo identifikuoti kaip geriausi hibridiniai palikuonys tolesnei selekcijai. Tyrimo metu gauti rezultatai padès atrinkti produktyvias $B$. rapa linijas selekcinėms programoms, vykdomoms heterozės kryptimi.

Reikšminiai žodžiai: heterobeltiozè, heterozè, lapų plaukuotumas, sèklų forma, sèklų spalva. 